\title{
Multiple Incremental Themes and Figure/Path Relations
}

\author{
John Beavers \\ The University of Texas at Austin
}

\section{Introduction}

Since Vendler (1957) it has been well known that the aspectual properties of dynamic predicates are partly determined by the choice of head verb. For example, the following verbs tend to head predicates of different aspectual classes:

a. build, destroy tend to head accomplishments (telic, durative).

b. laugh, walk tend to head activities (atelic, durative).

c. die, notice tend to head achievements (telic, punctual).

d. cough, tap tend to head semelfactives (telic, punctual or atelic, durative).

However, certain arguments in the predicate also enter into aspectual composition and partly determine the aspectual class of the predicate (Garey 1957, Verkuyl 1972, 1993, Tenny 1987, 1992, 1994, Krifka 1989, 1992, 1998, Dowty 1991, Jackendoff 1996). For example, for creation/consumption predicates (eat, drink, build) the expression of the patient argument has an effect on the aspectual class of the predicate. When the patient is expressed as a DP that has quantized reference (i.e. describes a specific quantity of the patient such that no subpart of the patient also has that quantity), then all else being equal the predicate is telic. If the patient is instead expressed as a DP that does not have quantized reference, or is omitted altogether, the predicate is atelic. This is shown in (2), where a glass of wine has quantized reference (no subpart of a glass of wine is also a glass of wine), and the predicate is telic, while wine does not have quantized reference (subparts of wine are still wine) and the predicate is atelic. I probe for telicity using the standard in and for temporal modifiers (Dowty 1979). Only telic predicates are compatible with in-modifiers, while atelic predicates are more natural with for-modifiers.

a. John drank a glass of wine in/?for an hour.

b. John drank (wine) for/??in an hour.

Likewise, for motion predicates, a bounded path expression (that specifies source and goal locations) can make the predicate telic as in (3a), while unbounded or omitted path expressions make the predicate atelic as in (3b).

(3) a. Dave walked from the university to the capital in/?for an hour.

b. Dave walked (towards the capital) for/??in an hour.

I call any argument that enters into aspectual composition in this way an "incremental theme", following Dowty (1991). ${ }^{1}$

\footnotetext{
${ }^{1}$ Tenny $(1987,1992,1994)$ uses this term only for patients of creation/consumption predicates.
} 
In his influential work on telicity, Krifka $(1989,1992,1998)$ analyzes incremental theme effects as a transfer of reference properties: how specific the predicate is about the quantity of the incremental theme determines how specific it is about the quantity of the event. Intuitively, in (2a) the drinking progresses as the agent progresses through the wine. Since we know how much wine there is, we know how much drinking there is. In (2b) the predicate does not say how much wine there is, and thus it does not say how much drinking there is. Similarly, in (3a) the walking progresses along the path from the university to the capital. Since we know where the path ends we know where the walking ends. In (3b) the predicate is not specific about how much path there is, and thus it is not specific about how much walking there is. Krifka (1998) proposes a series of homomorphic $\theta$-relations that relate events and incremental themes such that constraints on the mereological properties of one are reflected in the other in a way that preserves quantity and endpoints, giving us these correlations.

However, it is seldom noted (though see Dowty 1979: 63, Jackendoff 1996: 340-1, Filip 1999: 100-1, Rothstein 2004: 99) that some predicates appear to have multiple incremental themes. Consider again motion predicates. While unbounded paths determine atelicity, figure arguments that lack quantized reference also determine atelicity, even if the path is bounded. This is shown in (4) (adopted from Filip 1999: 100, (33)), where (4a) is telic and has both a figure with quantized reference and a bounded path expression. But the same predicate with a figure DP with nonquantized reference, an omitted path PP, or both is atelic, as in (4b-d) respectively.
a. The earthquake shook a book off the shelf in/?for a few seconds.
b. The earthquake shook books off the shelf for/??in a few seconds.
c. The earthquake shook a book for/??in a few seconds.
d. The earthquake shook books for/??in a few seconds.

However, the event does not simply stand in two separate homomorphisms. In this paper I show that the aspectual effects of each incremental theme are in fact dependent on the other: a bounded path expression is only relevant for telicity if the predicate says how much of the figure moves across the path, and likewise a figure expression with quantized reference is only relevant if the predicate says exactly where the figure moves to. I thus extend the model of telicity in Krifka (1998) to include a class of ternary $\theta$-relations that allow for double, interdependent incremental themes. In $\S 2$ I give more proper definitions of the relevant, previously proposed homomorphic $\theta$-relations for paths and theme arguments. In $\S 3$ I explore in more detail why these standard homomorphic relations do not account for predicates that have double incremental themes, focusing on motion. I also motivate the need for homomorphic relations more generally, focusing on the role they play in durativity. In $\S 4$ I propose a ternary $\theta$-role analysis of motion predicates in terms of a Figure/Path Relation (FPR), which decomposes an event by both the figure and the path, where the constraints imposed on each make reference to the other. In $\S 5$ I extend this to change-of-state and creation/consumption predicates, which I show also have double incremental themes. I conclude in $\S 6$. 


\section{Predicate Classes and Incremental Themes}

Classic incremental themes are objects of consumption predicates like (2), where progress of the event is tied isomorphically to the extent of the theme (Verkuyl 1972, 1993, Dowty 1979, 1991, Tenny 1987, 1992, 1994, Jackendoff 1996). For example, in (2) each part of the event corresponds to some part of the wine, and the event ends when the wine ends and vice versa. In this case the homomorphism $\theta$, which Krifka (1998: 213, (51)) calls a strictly incremental relation (SINC), preserves number of parts, i.e. a SINC has the properties in (5). ${ }^{2}$ For these definitions I assume a semantic model in which all entities fall into domains of objects $U_{P}$, events $U_{E}$, directed paths $U_{H}$, and/or connected direct paths $P_{H}$ and form mereological part/whole structures such that for any entities $x, x^{\prime} \in U_{X}, x^{\prime}$ may be a subpart of $x\left(x^{\prime}<_{X} x\right)$, a subpart or equal to $x\left(x^{\prime} \leq_{X} x\right)$, overlap with $x\left(x^{\prime} \circ_{X} x\right)$, or may be wholly disjoint from $x$. The sum (or join) of $x$ and $x^{\prime}$ is $x \oplus_{X} x^{\prime}$, and if $x^{\prime}<_{X} x, x \ominus x^{\prime}$ is the difference (the "left over part") of $x$ minus $x^{\prime}$. Finally, $x$ and $x^{\prime}$ may also be adjacent to one another $\left(x \infty_{X} x^{\prime}\right)$ in terms of some ordering relation. (See Krifka 1998 for more details; for expository purposes I occasionally omit subscripts if they are clear from context).

Strictly Incremental Relation (SINC): $e$ is $\theta$-related to patient $x$ such that every unique part of $e$ corresponds to a unique part of $x$ and vice versa. Thus $\theta$ is a SINC if it has the MUSO and MUSE properties: ${ }^{3}$

a. Mapping-to-unique-subobjects (MUSO):

$\forall x \in U_{P} \forall e, e^{\prime} \in U_{E}\left[\theta(x, e) \wedge e^{\prime}<_{E} e \rightarrow \exists ! y\left[y<_{P} x \wedge \theta\left(y, e^{\prime}\right)\right]\right]$

"For all $x \theta$-related to $e$, for all $e^{\prime}<e$ there is a unique $\theta$-related $x^{\prime}<x$."

b. Mapping-to-unique-subevents (MUSE):

$\forall x, y \in U_{P} \forall e \in U_{E}\left[\theta(x, e) \wedge y<_{P} x \rightarrow \exists ! e^{\prime}\left[e^{\prime}<_{E} e \wedge \theta\left(y, e^{\prime}\right)\right]\right]$

"For all $e \theta$-related to $x$, for all $x^{\prime}<x$ there is a unique $\theta$-related $e^{\prime}<e$."

For our purposes, a predicate is telic iff (6) holds, so that for any event it describes it does not describe any strict subpart of that event. ${ }^{4}$

$\forall X \subseteq U_{E}\left[T E L_{E}(X) \leftrightarrow \forall e, e^{\prime} \in U_{E}\left[X(e) \wedge X\left(e^{\prime}\right) \wedge e^{\prime} \leq_{E} e \rightarrow e=e^{\prime}\right]\right]$

" $X$ is telic if no $e$ in its denotation has a proper subpart in its denotation."

On the basis of the SINC in (5) and $\mathrm{TEL}_{E}$ in (6), the telicity facts for creation/consumption predicates follow. For example, (7) is predicted to be telic: ${ }^{5}$

\footnotetext{
${ }^{2}$ This is a strict incremental relation because it is isomorphic in number of subparts of the event and the theme. Krifka also defines a general incremental relation which permits more parts of the event than parts of the theme, e.g. in John read the book John may reread some parts of the book at different parts of the event. This is just a weakening of a SINC (or more propertly it embeds a SINC). For the simplicity I focus only on SINCs, though nothing hinges crucially on this.

${ }^{3}$ These properties are a collapsing of Krifka's (1998: 211-2) mapping-to-objects, mapping-toevents, uniqueness-of-objects, and uniqueness-of-events.

${ }^{4}$ Krifka (1998: 207, (37)) argues for a more sophisticated definition of telicity based on initial and final subparts. The advantages of this are irrelevant here, so I stick to the looser definition in (6).

${ }^{5}$ The $\lambda$-term represents the compositional meaning prior to existential generalization over the event variable. I ignore tense and the denotation of the temporal modifiers, which I only give to
} 
(7) Caesar drank two beers in/?for two hours.

$\lambda e \exists b\left[\operatorname{drink}^{\prime}\right.$ (caesar, $\left.b, e\right) \wedge 2$ beers $\left.^{\prime}(b)\right]$

For any event $e$ of drinking two beers $b$, any subevent of $e^{\prime}<e$ is an event of drinking some $b^{\prime}<b$ by the SINC relation that holds between $e$ and $b$. However, two beers has quantized reference, and therefore no such $b^{\prime}$ also satisfies this description (i.e. 2 beers ${ }^{\prime}\left(b^{\prime}\right)$ is false). This means $e^{\prime}$ cannot satisfy (7) since it is an event of drinking less than two beers. Thus for any $e$ satisfying (7) no $e^{\prime}<e$ also satisfies (7), and therefore (7) satisfies the definition of telicity in (6) and is correctly predicted to be telic. On the other hand, (8) is predicated to be atelic:

Caesar drank beer for/??in two hours.

$\lambda e \exists b\left[\operatorname{drink}^{\prime}\right.$ (caesar, $\left.\left.b, e\right) \wedge \operatorname{beer}^{\prime}(b)\right]$

For any event $e$ of drinking some amount of beer $b$, any subevent $e^{\prime}<e$ is an event of drinking some $b^{\prime}<b$. However, beer does not have quantized reference, so that any $b^{\prime}<b$ still satisfies this description. ${ }^{6}$ Thus $e^{\prime}$ (an event of drinking beer) will satisfy (8), and therefore since (8) can describe both $e$ and $e^{\prime}$ it does not satisfy (6) and is predicted to be atelic. The relevant transfer of reference properties is in the number of parts: the event and the incremental theme are isomorphic in this regard, and constraints the predicate imposes on the incremental theme regarding the number of subparts determine the interpretation of the event.

Paths of motion are also incremental themes (Tenny 1987, 1992, 1994, Dowty 1991, Jackendoff 1996, Krifka 1998). Here the relevant $\theta$-relation is a strict movement relation (SMR) (Krifka 1998: 224, (68)). An SMR preserves number of parts, just as the SINC does, but also preserves spatial/temporal adjacency: progress of the event involves progress along the path, where temporally adjacent progress through the event corresponds to spatially adjacent progress along the path. For example, in John walked from home to the store the event begins when John leaves home, progresses temporally as John progresses spatially along the path, and ends when John reaches the store. This relation is given in (9). ${ }^{7}$

(9) Strict Movement Relation (SMR): $e$ is $\theta$-related to the path $p$ such that every unique part of $e$ is $\theta$-related to a unique part of $p$ and vice versa, where temporal adjacency in $e$ corresponds to spatial adjacency in $p$ and vice versa. Thus $\theta$ has the $\mathrm{ADJ}, \mathrm{MO}$, and $\mathrm{CP}$ properties:

a. Adjacency (ADJ):

$\forall x, y, z \in P_{H} \forall e, e^{\prime}, e^{\prime \prime} \in U_{E}\left[\theta(x, e) \wedge e^{\prime}, e^{\prime \prime} \leq_{E} e \wedge y, z \leq_{H} x \wedge \theta\left(y, e^{\prime}\right) \wedge\right.$ $\left.\theta\left(z, e^{\prime \prime}\right) \rightarrow\left[e^{\prime} \infty_{E} e^{\prime \prime} \leftrightarrow y \infty_{H} z\right]\right]$

demonstrate (a)telicity. For expository purposes I use a standard $n$-ary notation for the denotation of verbal lexemes, but I nonetheless presuppose an underlying set of Parsons (1990) style $\theta$-relations. I assume definite, specific DPs have direct reference, and I represent constants in boldface.

${ }^{6}$ Rather, beer has divisive reference (any subpart of beer is still beer) and cumulative reference (the join of two items in the denotation of beer is also in the denotation of beer) (Kiparsky 1998).

${ }^{7}$ Just as Krifka proposes a general incremental relation, he also proposes a general movement relation that embeds an SMR but allows backtracking, pauses, and loops. Again, nothing hinges on this here so I focus only on the SMR. 
"For $\theta$-related $e$ and $x$, for any $y, z \leq_{H} x \theta$-related to $e^{\prime}, e^{\prime \prime} \leq_{H} e$ respectively, $y$ is spatially adjacent to $x$ iff $e^{\prime}$ is temporally adjacent to $e^{\prime \prime}$."

b. Mapping-to-objects (MO):

$\forall x \in U_{P} \forall e, e^{\prime} \in U_{E}\left[\theta(x, e) \wedge e^{\prime} \leq_{E} e \rightarrow \exists y\left[y \leq_{P} x \wedge \theta\left(y, e^{\prime}\right)\right]\right]$

"For all $x \theta$-related to some $e$, for all $e^{\prime}<e$ there is a $\theta$-related $x^{\prime}<x$."

c. Movement happens along connected paths (CP):

$\forall x \in U_{H} \forall e \in U_{E}\left[\theta(x, e) \rightarrow x \in P_{H}\right]$

"For all $x \theta$-related to any $e, x$ is part of a connected path structure."

The MO property ensures that each part of the event is mapped to some part of the path, where the ADJ and $\mathrm{CP}$ properties ensure that connected, adjacent temporal progress corresponds to connected, adjacent spatial progress. An SMR is therefore an isomorphism of number of parts and adjacency, and the telicity of a motion predicate is keyed to the expression of its path. For example, (10) is predicted to be telic since the path is clearly delineated as being from the jar to the floor.

(10) The carafe of wine flowed from the jar to the floor in/?for five minutes. $\lambda e \exists p\left[\right.$ flow $^{\prime}($ carafe, $p, e) \wedge S O U R C E($ jar $, p, e) \wedge G O A L($ floor $\left., p, e)\right]$

This follows from the definition of an SMR in (9) and telicity in (6). For any event $e$ satisfying (10), any $e^{\prime}<e$ is a subevent of flowing along some subpath $p^{\prime}<p$. However, if $p^{\prime}$ is a subpath of a path from the jar to the floor it is not itself a path from the jar to the floor. It is a path that excludes the jar, the floor, or some connecting part in between. Thus $e^{\prime}$ cannot satisfy (10) since $p^{\prime}$ will either not satisfy both the SOURCE and GOAL conditions or it will violate the CP and ADJ properties. Therefore no subevent of $e$ satisfies (10), which therefore satisfies (6) and is predicted to be telic. Conversely, (11) is predicted to be atelic.

(11) The carafe of wine flowed for/??in five minutes.

$$
\lambda e \exists p \exists x \exists y\left[\text { flow }^{\prime}(\text { carafe, }, p, e) \wedge \operatorname{SOURCE}(x, p, e) \wedge \operatorname{GOAL}(y, p, e)\right]
$$

For any event $e$ matching (11) any $e^{\prime}<e$ is a subevent of flowing along some $p^{\prime}<p$. However, (11) imposes no constraints on the path save that it has some beginning and ending, so that any such $p^{\prime}$ vacuously satisfies these constraints. Thus $e^{\prime}$ satisfies (11), which means (11) does not satisfy (6) and thus is atelic. For other classes of predicates other homomorphisms may be relevant (though I argue below that all dynamic predicates entailing change involve at least one $\theta$-relation in common). The crucial point is that on Krifka's model the event is always related to some argument in some way that generates a transfer of reference properties: certain mereological properties the predicate imposes on the incremental theme result in corresponding mereological properties imposed on the event, which telicity is crucially tied to.

\section{Double Incremental Themes in Motion Predicates}

However, there are predicates that have two incremental themes, i.e. two arguments that enter into the aspectual composition. In this section and the following I fo- 
cus on motion, though in $\S 5$ I expand this discussion to change-of-state and creation/consumption as well. In (12), a figure argument with non-quantized reference can determine that the motion predicate is atelic even if the path is fully bounded.

(12) Wine flowed from the jar to the floor for/??in five minutes.

$\lambda e \exists p \exists w\left[\operatorname{flow}^{\prime}(w, p, e) \wedge\right.$ ine $^{\prime}(w) \wedge \operatorname{SOURCE}(\mathbf{j a r}, p, e) \wedge G O A L($ floor $\left., p, e)\right]$

The atelicity of (12) is surprising since the constraints imposed on the path (the incremental theme on an SMR approach) are identical to those in (10), which is telic. Thus (12) should be telic for exactly the same reason (10) is. Yet it is atelic, suggesting that the figure is an incremental theme. How can we explain this?

We could assume that there are simply two homomorphisms here. In other words, suppose the event stands in an SMR with the path and a SINC with the theme simultaneously. However, this cannot be, since imposing both constraints simultaneously leads to contradictions. First, in a motion event like (12) it could be the case that different proper parts of the wine are moving at the same point in the event. For example, suppose the wine in (12) is gushing out a few globules at a time. It is entirely possible that there could be several pools of wine moving independently, so that for example one pool hits the floor while another leaves the jar at exactly the same time. This putatively violates the SINC, where by the MUSE property in (5b) each subevent correspond to a unique part of the figure. In this case a particular subevent corresponds to two parts of the figure, a contradiction to the SINC. However, one could argue that these two parts of the figure mereologically form a single part of the figure (since the SINC says nothing about spatial contiguity of the incremental theme). This reconceptualization would not be a violation of the SINC, since now only one part of the wine is moving at that point in the event, albeit a "part" of the wine that consists of two spatially disjoint subparts. However, this reduction at least intuitively misses the fact that the wine is perceptually subdivided into two distinct subparts moving simultaneously, something we should expect a linguistic description of such an event to be able to accommodate.

More problematically, it is also possible that non-adjacent subpaths of the entire path can be crossed at temporally adjacent portions of the event, which violates the SMR. For example, suppose the path from the jar to the floor contains of a long stretch along a table. It is possible in (12) that at some time $t$ some part of the wine reaches the floor, while at $t+1$ some (other) part of the wine flows out of the jar onto the table. Here we have two temporally adjacent periods of time, but they do not correspond to spatially adjacent movement since the movement at $t$ happens in a location wholly unconnected to where the movement at $t+1$ occurs. This, however, would violate the SMR, where temporally adjacent traversal must be along adjacent subpaths. Thus certain situations which conform naturally to one homomorphism create contradictions (or at least perceptual complications) for the other, such that it cannot be that two homomorphisms hold at the same time.

Alternatively, we could assume that there is only one homomorphism, and explain the effects of the other argument in some other way (as proposed by Rothstein 2004, who eliminates homomorphisms between events and themes). We could even assume that no homomorphism exists at all, and that telicity altogether is de- 
termined in some other way (as is implicit in Hay et al. 1999 and Kennedy and Levin 2008). However, there is reason to believe that mereological properties of both figures and paths are relevant for the aspectual properties of the predicate. In particular, Wechsler $(2001,2003,2005)$ and Beavers $(2002,2008 b)$ show that the mereological complexity of paths affects the durativity of the predicate. Looking again at motion predicates, if the path is simplex (transitional), the predicate is punctual. If the path is complex, the predicate is durative. This is shown in the contrast between a transitional border vs. a complex path in (13). I test for durativity again with in-modifiers, which have after readings with punctual predicates but have both after and during readings with durative predicates (Kearns 2000). ${ }^{8}$
a. The settler will cross the border in ten days.
(after; punctual)
b. The settler will cross the desert in ten days.
(after/during; durative)

In Beavers $(2002,2008 b)$ I suggest that there are two linguistically relevant mereological types for paths and events. A path or event may have two parts, i.e. a beginning and an end (it is bipartite, a Minimally Complex Object), or it may have more than two elements, i.e. a beginning, a middle, and an end (it is > bipartite, a Complex Object). A minimally complex (bipartite) path is transitional, and a complex path (>bipartite) has multiple transitions. Likewise, a minimally complex (bipartite) event is punctual, and a complex (>bipartite) event is durative. On this classification scheme, the following generalization emerges from (13):

a. Simple path (bipartite) $\Leftrightarrow$ punctual event (bipartite).

b. Complex path (>bipartite) $\Leftrightarrow$ durative event (>bipartite).

However, something not discussed in my earlier work is that it is also crucial in (13) that the figure is mereologically atomic. If we instead have a mereologically complex figure (such as two or more settlers) it is possible to have a durational reading as well, even if the path is simple (bipartite):

$$
\begin{aligned}
& \text { a. The settlers will cross the border in ten days. } \\
& \text { b. The settlers will cross the desert in ten days. }
\end{aligned}
$$

The relevant context for (15a) is that the settlers are crossing the border slowly, one by one, for ten days. Thus the internal complexity of the figure is also relevant for durativity, where complex figures allow (though do not require) durative readings.

Therefore the mereological complexity of both arguments figures in the mereological complexity of the event. This is by definition homomorphic (property preservation), and at least partly follows from the discussion above. As discussed by Beavers $(2002$, 2006, 2008b), a generalized movement relation based on the SMR in (9) (see fn. 7) determines a preservation of up to tripartite complexity between paths and events. Roughly, a transition from one point to another on a path corresponds to a two place event, but if the motion event is longer than this there must

\footnotetext{
${ }^{8}$ For some reason this test produces the clearest results with a modal or temporal auxiliary, although the judgments also obtain in the simple past. The in-test only applies to telic predicates. The test for atelic predicates is acceptability with for-modifiers (cf. John jogged for an hour/\#blinked once for an hour).
} 
be some additional piece of path the motion occurred on. If the path is longer than two points there must be some additional portion of the event beyond the beginning and the end corresponding to the figure traversing this extra piece of the path. So (14) follows from an SMR-type relation (see Beavers 2008b for a more detailed discussion). ${ }^{9}$

Although I have not analyzed the facts in (15) in my previous work, intuitively something similar holds. I assume that all paths are minimally bipartite (since an atomic path is just a location). For a bipartite path, if the figure is atomic, it simply transitions from one point on the path to the other, yielding a punctual event as in (14). But if the figure is non-atomic, then it is possible to break down the event into several events of each part of the figure transitioning along the path. This involves more than one punctual event, and the sum of multiple punctual events is a durative event (it has more than two parts), yielding durativity. Of course, if the path is complex the event will be durative regardless of the figure. Thus for both arguments the relation is clearly homomorphic (complexity preservation), suggesting that we cannot simply eliminate homomorphisms from our approach to avoid the contradictions possible with (12). But how can we analyze (12) homomorphically in a way that does not yield a contradiction?

\section{Figure/Path Relations}

I show that we can resolve this problem via a more complex mapping between the event and the two arguments. The crucial issue in (12) is one of relativity. Different parts of the figure may move simultaneously in an event (contra the SINC), but only on different parts of the path. Mereologically, any two subparts of the figure moving along the exact same part of the path must be the same part of the figure. Likewise, non-adjacent parts of the path may be traversed at temporally adjacent parts of the event (contra the SMR), but only by different parts of the figure. It is impossible for the same part of the figure to traverse spatially non-adjacent parts of the path at temporally adjacent times (barring telekinesis). This suggests a more complex interaction between the event and the two arguments: the constraints stated on each make reference to the other. Therefore, just as there is no notion of a moving figure without a path it moves along nor vice versa, I suggest that we cannot define a homomorphic $\theta$-relation for a figure without making reference to a path and vice versa.

Indeed, the one attempt at a treatment of double telicity that I am aware of is the Conceptual Semantics sketch of Jackendoff (1996: 344), which involves two separate "dimensions" of the event, each mapped to a different argument by a different homomorphism (what he calls a "structure-preserving binding"). This creates what he refers to as two different "perspectives" on the event: one of each

\footnotetext{
${ }^{9}$ This correlation also explains a range of other facts, including constraints on possible resultatives and the interpretation of change-of-state predicates in different contexts. I discuss this further in $\S 5$ (see Beavers 2006: Ch.4 for a summary of this approach).
} 
part of the path being crossed by all of the figure, the other of a part of the figure crossing all of the path. Therefore this analysis has the relevant notion of relativity built into it, although Jackendoff never discusses the contradictions in $\S 3$, nor the necessity of stating constraints on themes and paths relative to one another. However, his analysis is problematic, since it is not conceptually obvious what it means for an event to have several temporal dimensions (since time is one-dimensional, as he acknowledges; ibid.: 345), nor is there any linguistic motivation for saying events are multi-dimensional in this way. Following the formalization of Krifka, I give an analysis that avoids the two-dimensional issue by looking instead at different mereological breakdowns of the same event.

I propose that motion is an inherently three-place, mutually-constraining relation between a figure $x$, a path $p$, and an event $e$. A motion event can be decomposed into a series of motion subevents, each of which corresponds to some part of $x$ crossing all of $p$ (following Filip 1999: 141-2 on multiplicity of events). For example, (12), repeated here, can be decomposed into events of each part of the wine (perceptual, conceptual, or actual) traversing the path.

Wine flowed from the jar to the floor for/??in five minutes.

$$
\lambda e \exists p \exists w\left[\text { flow }^{\prime}(w, p, e) \wedge \text { wine }^{\prime}(w) \wedge \operatorname{SOURCE}(\mathbf{j a r}, p, e) \wedge G O A L(\text { floor }, p, e)\right]
$$

The cumulative effect is that all of the wine crosses all of the path. I refer to this as a Figure/Path Relation (FPR), a ternary $\theta$-role that I define recursively as follows:

A relation $\theta$ is an FPR iff for every $e, x, p$ such that $\theta(e, x, p)$ :

a. $e$ and $p$ stand in an SMR or

b. there exists $x^{\prime}<x$ for which there exist a unique $e^{\prime}<e$ such that $e^{\prime}$ stands in an SMR to $p$ and the remainder of $x$ and $e$ stand in an FPR with $p$, i.e. $\exists x^{\prime} \exists ! e^{\prime}\left[x^{\prime}<x \wedge e^{\prime}<e \wedge S M R\left(\left\{<e^{\prime}, p>\right\}\right) \wedge F P R\left(\left\{<e \ominus e^{\prime}, x \ominus x^{\prime}, p>\right\}\right)\right]$.

Thus for each $x_{i}<x(1 \leq i \leq n)$, an FPR "slices off" a unique motion subevent $e_{i}<e$ of $x_{i}$ crossing $p\left(e_{i}\right.$ stands in an SMR with $p$ ). The rest of $e$ and $x$ stand recursively in an FPR with $p$, until $e$ is all carved up. This preserves number of subparts between $x$ and $e$ up to individual motion subevents. I represent this visually as follows:

The event:

For $x_{i}<x, p$ constant:

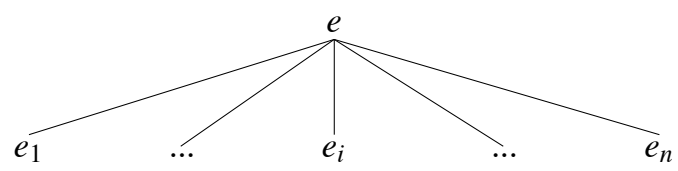

This reproduces the isomorphism in the SINC, except that the "smallest" events here are still complex motion events. This breakdown captures the atelicity of (16). For event $e$ described by (16) of some wine $w$ crossing a path $p$, any subpart $w_{i}<w$ corresponds to some $e_{i}<e$ that is SMR-related to $p$ by the FPR, corresponding to $w_{i}$ crossing $p$. Since $w_{i}$ is still wine, $e_{i}$ satisfies (16). Since (16) describes both $e$ and $e_{i}$ it is atelic by the definition of telicity in (6).

Such cases are relatively straightforward. But do we still get the atelicity of examples like (19) with unbounded paths?

(19) The carafe of wine flowed for/??in one minute.

$$
\lambda e \exists p \exists x \exists y\left[\text { flow }^{\prime}(\text { carafe }, p, e) \wedge \operatorname{SOURCE}(x, p, e) \wedge \operatorname{GOAL}(y, p, e)\right]
$$


In fact we do, in a way similar to the SMR. To illustrate this I first discuss how we can further break down each one of these motion subevents in (18). For all relevant $x_{i}$, the FPR predicts there is a motion subevent $e_{i}$ of $x_{i}$ crossing $p$. This means that $e_{i}$ stands in an SMR with $p$. However, if $e_{i}$ stands in an SMR with $p$ and SMRs decompose events by parts of the path, we can also identify a unique subevent $e_{i}^{j}<e_{i}$ for each $p^{j}<p(1 \leq j \leq m)$. I represent this visually as follows:

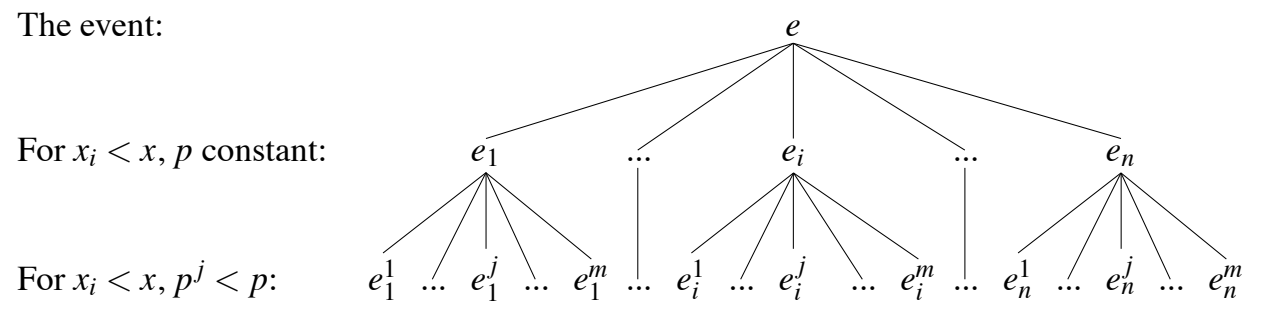

For all $i, j, e_{i}^{j}$ corresponds to $x_{i}$ crossing $p^{j}$. Thus we have first broken down $e$ in terms of individual motion subevents for each subpart of $x$, and since each of these motion subevents is a motion event of the usual sort, we can break each down further according to subparts of the path, giving us a series of subevents of $e$ each representing a single part of $x$ crossing a single part of $p$.

Now, if we hold a given subpath $p^{j}<p$ constant, we can identify all of the subevents in this breakdown corresponding each $x_{i}<x$ crossing $p^{j}$. For example, for $x=x_{1} \oplus \ldots \oplus x_{i} \oplus \ldots \oplus x_{n}$ there are subevents $e_{1}^{j}, \ldots, e_{i}^{j}, \ldots, e_{n}^{j}$ of each piece of $x$ crossing $p^{j}$. The sum of all of these subevents is $e^{j}=\sum_{i=1}^{n} e_{i}^{j}$, corresponding to all of $x$ crossing $p^{j}$, since it is the sum of the subevents of each piece of $x$ crossing $p^{j}$. In a sense we have taken the breakdown represented in (20) and rearranged the smallest subevents so that instead of being grouped first by subparts of $x$ they are grouped first by subpaths of $p$, and then joined for each $p^{j}$, represented as follows:

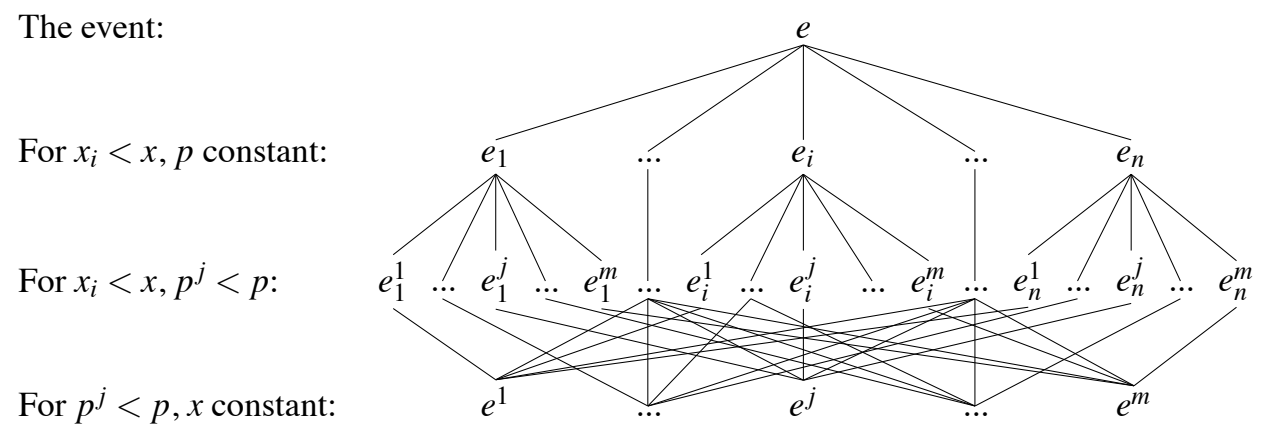

Each of these subevents is an event of all of $x$ crossing a different part of $p$. But of course, if we sum all of these events together, the result is simply $e=\sum_{j=1}^{m} e^{j}$, the entire event. This preserves number of subparts and adjacency between $e$ and $p$ up to the motion of each part of the figure, mirroring the SMR. I represent this visually as follows: 


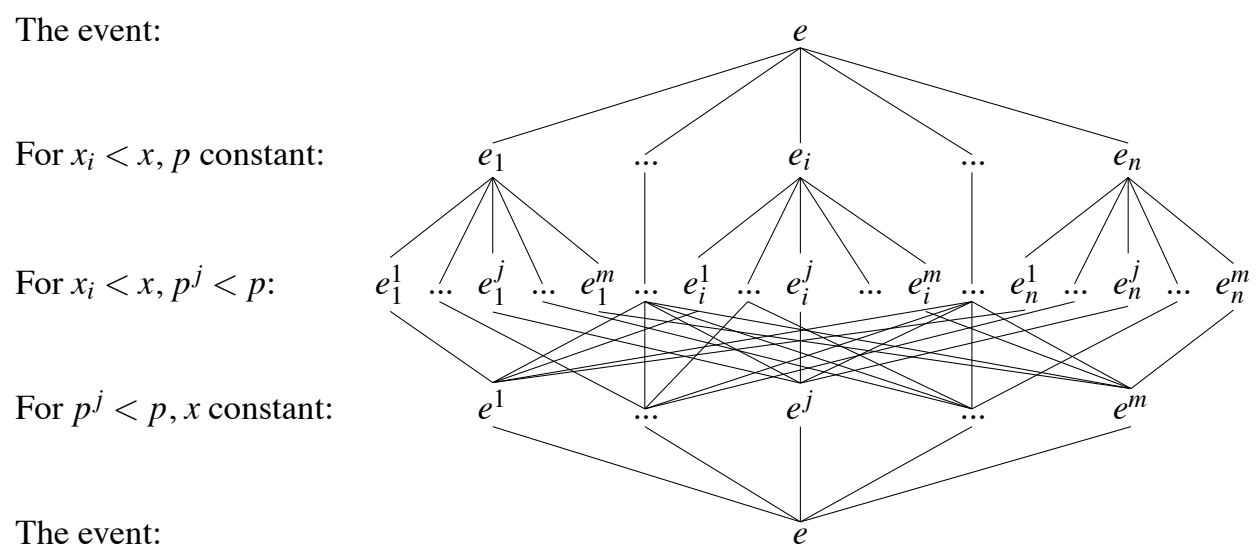

Thus like Jackendoff's analysis, the FPR represents multiple decompositions of (or perspectives on) the event: one by the subparts of the figure keeping the path fixed, another by subparts of the path keeping the figure fixed, and the third a criss-crossing of the two. However, unlike Jackendoff, the event is temporally onedimensional. The different perspectives correspond instead to different ways of carving up the event. Furthermore, the criss-crossing perspective provides a way of linking the other two perspectives up by further decomposition and reorganization, something not explicit on Jackendoff's approach. Indeed, this middle breakdown of the event is the heart of the FPR, and defines the FPR as a set of all unique ordered triples relating the breakdown of the event, the path, and the figure together.

The atelicity of (19), repeated here, follows from this, in particular from the breakdown represented in the last line in (21).

(23) The carafe of wine flowed for/??in one minute.

$$
\lambda e \exists p \exists x \exists y\left[\text { flow }^{\prime}(\text { carafe, }, p, e) \wedge \operatorname{SOURCE}(x, p, e) \wedge \operatorname{GOAL}(y, p, e)\right]
$$

For any $e$ satisfying (23) for path $p$, there is some $e^{j}<e$ corresponding to $p^{j}<p$ by the FPR. But any such subpath $p^{j}$ satisfies the (lack of) constraints in (23), since all (23) says about the path is that it must have a beginning and an end (true of all paths). Thus (23) describes both $e$ and $e^{j}$, and is therefore atelic. Of course, if the figure has non-quantized reference and the path is unbounded, we also get atelicity:

Wine flowed for/??in one minute.

$$
\lambda e \exists p \exists w \exists x \exists y\left[\operatorname{flow}^{\prime}(w, p, e) \wedge \operatorname{wine}^{\prime}(w) \wedge \operatorname{SOURCE}(x, p, e) \wedge \operatorname{GOAL}(y, p, e)\right]
$$

For any $e$ described by (24) for some wine $w$ and path $p$, any subevent $e^{\prime}<e$ corresponds either to some $w^{\prime}<w$ crossing $p$ or $w$ crossing $p^{\prime}<p$ or both by the FPR. But any $w^{\prime}$ or $p^{\prime}$ will satisfy the constraints in (24), and thus so will $e^{\prime}$, meaning the predicate is atelic. On this analysis, the only way for a motion predicate to be telic is if quantization/boundedness constraints are placed on both the figure and path, correctly predicting that only (25) is telic.

(25) The carafe of wine flowed from the jar to the floor in/?for one minute. $\lambda e \exists p[\operatorname{flow}($ carafe $, p, e) \wedge \operatorname{SOU} R C E($ jar $, p, e) \wedge G O A L($ floor $, p, e)]$

For any $e$ described by (25) for some wine $w$ and path $p$, any $e^{\prime}<e$ corresponds to either $w^{\prime}<w$ crossing $p$ or $w$ crossing $p^{\prime}<p$ or both by the FPR. In this case, no 
$w^{\prime}$ or $p^{\prime}$ will match the constraints imposed by (25). Thus $e^{\prime}$ is not in the denotation of (25) and (25) is therefore telic. This crucially requires a $\theta$-relation in which constraints on each argument are stated dependent on the others. Telicity therefore reduces not simply to all of the figure moving or all of the path being crossed, but of all of the figure crossing all of the path. For this to be known it must be that the predicate indicates both how much figure there is and where the path ends.

\section{Unifying the Predicate Classes}

This model has wider application than just motion. In Beavers (2008b) I argue that motion relations can be used to model the underlying semantics of all types of dynamic predicates that entail changes, including change-of-state and consumption predicates. However, this earlier work focuses primarily on the facts discussed in $\S 3$ that durativity correlates with the complexity of the change involved, where complexity of change was modeled in terms of complexity of an abstract path argument representing the scale of change. In Beavers (2006, 2008c) I further support this analysis by looking at the different types of semantic contrasts found in various sorts of object/oblique alternations. I show that a wide range of object/oblique alternations can be swept under a simple analysis where the relevant semantic contrast involves increasingly more specific entailments about the endpoints of an abstract path, in a way that supports giving a unified semantics to a range of dynamic predicates. In this section I further support an analysis of change-of-state and creation/consumption predicates as having a similar underlying semantics to motion predicates by showing that we also find double incremental theme effects with them as well, thus arguing not only that they all share a common underlying semantics (as proposed in my earlier work), but also that this semantics involves an underlying ternary $\theta$-role, in particular an FPR (expanding my earlier work).

First and foremost, change-of-state predicates are often analyzed as motion along an abstract path/scale (Tenny 1987, 1992, 1994, Dowty 1991, Krifka 1998, Filip 1999, Jackendoff 1996, Hay et al. 1999, Wechsler 2001, 2003, 2005, Beavers $2002,2006,2008 b, c)$. In other words, we can loosely give the following analyses to wipe, blush, and polish, where each predicate $\phi$ describes an event $e$ in which some patient argument $x$ "moves" abstractly along some property scale $s$ until reaching some final goal state $g$ on some scale $s$ (where the predicate supplies the type of $s$ ):

a. Caesar wiped the table clean. (Progress of $e \Leftrightarrow$ cleanliness scale) $\lambda e \exists s\left[\right.$ wipe $^{\prime}(\mathbf{c a e s a r}$, table $, s, e) \wedge \operatorname{SOURCE}(\mathbf{d i r t y}, s, e) \wedge G O A L($ clean $\left., s, e)\right]$

b. Jane blushed a fierce red. (Progress of $e \Leftrightarrow$ redness scale) $\lambda e \exists s\left[b l u s h^{\prime}(\right.$ jane $, s, e) \wedge \operatorname{SOURCE}(\mathbf{n o r m a l}, s, e) \wedge G O A L($ fierce.red $\left., s, e)\right]$

c. Joe polished his shoes to a nice shine. (Progress of $e \Leftrightarrow$ shininess scale) $\lambda e \exists s\left[\right.$ polish $\left.^{\prime}(\mathbf{j o e}, \mathbf{s h o e s}, s, e) \wedge \operatorname{SOURCE}(\mathbf{d u l l}, s, e) \wedge \operatorname{GOAL}(\mathbf{s h i n e}, s, e)\right]$

On such an approach result XPs like clean, a fierce red, and to a nice shine supply endpoints on scales just as goal PPs supply endpoints on paths. In other words, 
result XPs and goal PPs are two manifestations of the same notion. Evidence for this comes from the fact that result XPs can also be PPs (as in (26c)), headed by the same prepositions that introduce goals (e.g. to, into). Furthermore, motion and change-of-state have similar effects on the durativity of the predicate. In particular, certain result XPs determine punctuality of the change-of-state predicate, while others determine durativity. This is shown in the contrast in (27), where shot the sheriff is punctual with the result XP dead but durative with the result XP to death.

a. Wyatt shot the sheriff dead in five minutes. (after; punctual)

b. Wyatt shot the sheriff to death in five minutes. (after/during; durative)

As shown by Wechsler $(2001,2005)$ and Beavers $(2002,2008 \mathrm{~b})$, this corresponds to the gradability of the scalar expression. The adjective dead is non-gradable, corresponding to a binary contrast between states not dead and dead, like pregnant and some prepositions such as on (Kennedy 2001, Beavers 2008b). Such expressions are unacceptable with comparative morphology. On the other hand, to-PPs pattern with gradable adjectives such as polished and clean, which are compatible with comparative morphology (Beavers 2008b: 251).

(28) a. Non-gradable (binary): he is dead, \#he is more dead, she is pregnant, \#she is more pregnant, the fly is on the wall, \#the fly is more on the wall.

b. Gradable (>binary): the shoes are clean, the shoes are cleaner/more clean, the shoes are polished, the shoes are more polished, this path goes to(ward) the lake, this path goes more/further to(ward) the lake.

The generalization emergent from (27) is that non-gradable XPs determine punctual predicates and gradable XPs determine durative predicates. ${ }^{10}$ The effect in (27) is thus exactly the effect seen in $\S 3$ for motion predicates, on the assumption that non-gradable XPs mark endpoints on bipartite scales and gradable XPs mark endpoints on > bipartite scales, just as different paths are bipartite or $>$ bipartite. Thus the durativity facts in (27) can be modeled simply in terms of a homomorphism between the property scale and the event, exactly as assumed for motion. Indeed, homomorphic effects can be seen even without overt scalar XPs (Beavers 2008b). Context can determine that certain types of changes are complex or simplex, which in turn has an aspectual effect on the predicate, as in the following example:

Wade turned off the lights. (punctual if by switch, durative if by knob)

This suggests that just as motion predicates have a covert path argument even when not overtly expressed (e.g. for John walks to be true he must have walked along some path), change-of-state predicates entail the existence of some scale of change

\footnotetext{
${ }^{10} \mathrm{~A}$ similar correspondence of gradability to durativity is seen in the mapping from adjectives to verbs, i.e. deadjectival verbs based on gradable adjectives tend to be durative, while those based on non-gradable adjectives tend to be punctual (Kennedy and McNally 2005):

(i) a. durative verb $\leftrightarrow$ gradable adjective $\quad\left(\right.$ clean $_{V} /$ clean $_{A}$, polish/polished)

b. punctual verb $\leftrightarrow$ non-gradable adjective

(die/dead, break/broken)
} 
even if not overt. Indeed, just as there is no notion of a figure without a path and vice versa, there is no patient without a transition along a measurable property and vice versa. All of this suggests (as Krifka 1998, Beavers 2008b propose) that the underlying thematic relation relating scales to events is just another type of movement relation, what Beavers (2008b) refers to as a Generalized Movement Relation (GMR) which has the same mereological preservation properties as a normal movement relation but which covers both physical paths and abstract scales.

Of course, on this analysis the telicity facts for change-of-state predicates can be modeled in the same way as with motion: boundedness of the scale yields telicity, and unbounded or omitted scales yield atelicity (Krifka 1998: 228-30). This is shown in (30), where an overt bound on the change (the result XP clean) determines telicity while an unbounded scalar (cleaner and cleaner; Goldberg and Jackendoff 2004) or an unexpressed result determine atelicity.

a. Caesar wiped the table clean (in/?for an hour).

$\lambda e \exists s\left[\right.$ wipe $^{\prime}($ caesar, table $, s, e) \wedge S O U R C E(\operatorname{dirty}, s, e) \wedge G O A L($ clean $\left., s, e)\right]$

b. Caesar wiped the table (cleaner and cleaner) (for/??in an hour).

$\lambda e \exists s \exists x \exists y\left[\right.$ wipe $^{\prime}$ (caesar, table, $\left.\left.s, e\right) \wedge \operatorname{SOURCE}(x, s, e) \wedge \operatorname{GOAL}(y, s, e)\right]$

However, following the discussion above, we may instead want to analyze this not in terms of a GMR but in terms of an FPR, which incorporates the same insights as GMR analysis but also predicts that the theme argument should also enter aspectual composition. We have evidence for this, since change-of-state predicates also show double incremental theme effects. Not only does the absence/unboundedness of a result XP determine atelicity, but so does a patient argument with non-quantized reference, as in (31).

(31) Caesar wiped tables clean for/??in an hour.

This suggests that an FPR operates here as well, though along a more abstract path. This provides still further evidence that the underlying aspectual structure of motion events and the underlying aspectual structure of change-of-state predicates are identical. Each involves some figure/patient changing along some scale, where for motion the scale is a physical path and for change-of-state it is an abstract scale.

However, the one predicate class least amenable to a scalar analysis are creation/consumption predicates (though see Hay et al. 1999, Wechsler 2005, Beavers 2002, 2006, 2008b,c). In these cases the older analysis (that the event is mapped only to the theme) seems best. In other words, in John drank wine the event seems to progress directly through the wine, not through some abstract property of the wine (such as its volume or existence). Evidence for the absence of an underlying scale comes from the fact that there are few appropriate result state modifiers to be found with such predicates, suggesting there is no underlying scale to modify:

a. John drank his beer up/?all gone/*gone/*to emptiness.

b. James ate that sandwich up/?all gone/*gone/*to non-existence.

Despite the obvious semantic plausibility of drinking something until it is gone, gone and similar modifiers are not fully acceptable with drink and eat (though completive up is fine, and all gone is a bit more natural than the rest, something I return 
to below). Likewise, it is certainly possible for mereologically simplex objects to yield punctual predicates, as shown in (33) for eat:
a. John ate a piece of popcorn in 37 minutes.
(after; punctual)

b. John ate a whole fried chicken in 37 minutes. (after/during; durative)

It is difficult to generate the same sort of contrast based on the underlying change. There are no simplex result XPs we can use to make the predicate punctual as in (27) (since there are no result state modifiers period), and it is hard to do the sort of recontextualization shown in (29) to get a punctual reading of John ate a whole fried chicken. So it does seem here that event is mapped only to the patient, and not to any other argument, i.e. here we have just a SINC and not an FPR.

However, there is evidence to suggest an FPR, in particular regarding the conative alternation in (34), where the patient of a creation/consumption verb can be marked by the preposition at, shown here for drink (similar results hold for eat).

a. Caesar drank a glass of beer.

b. Caesar drank at a glass of beer (slowly).

As Beavers $(2006,2008 \mathrm{c})$ argues in considerable detail, in (34b) the conative alternation has the effect of leaving unspecified the "complete" creation/consumption reading inherent to such predicates. The reading of the at variant is that some drinking occurred, but not necessarily a complete drinking event:

(35) a. Caesar drank a glass of beer, \#but didn't finish it.

b. Caesar drank at a glass of beer (slowly), but didn't finish it.

Crucially, the at variant in these cases is in fact atelic, despite having an incremental theme with quantized reference. This is shown in (36).

a. Caesar drank a glass of beer in/?for five minutes.

b. Caesar drank at a glass of beer (slowly) for/??in an hour.

If only the theme argument enters into aspectual composition, it is hard to explain the atelicity of (36). However, it is possible instead to analyze this as a predicate with an FPR as well, with the scale being one of existence, where telicity is partly determined by the boundedness of the scale. As Beavers $(2006,2008 \mathrm{c})$ proposes, the conative in (36) is an operation that produces an existential generalization over the endpoints of the scale, which for creation/consumption predicates are lexically specified by default to be "complete creation/consumption" as in (37). This gets not only the semantics right, but also gives us atelicity for free in the same way it is predicted for motion and change-of-state predicates.

a. Caesar drank his beer.

$\lambda e \exists s\left[\operatorname{drink}^{\prime}(\right.$ caesar, beer, $s, e) \wedge \operatorname{SOURCE}(\mathbf{f u l l}, s, e) \wedge G O A L($ empty, $\left.s, e)\right]$

b. Caesar drank at his beer.

$\lambda e \exists s \exists x \exists y\left[\operatorname{drink}^{\prime}\right.$ (caesar, beer, $\left.\left.s, e\right) \wedge \operatorname{SOURCE}(x, s, e) \wedge \operatorname{GOAL}(y, s, e)\right]$ 
The predicate in (37a) guarantees telicity, while (37b) does not. But why, if there is an underlying scale, are consumption predicates not amenable to result XP modification? This may be because the result state is already highly lexically specific: the default is complete creation/consumption. Rappaport Hovav and Levin (1998) note that in general result verbs (e.g. break) and manner (non-result) verbs (e.g. sweep) differ in terms of resultative predication. The more specific the result state, the fewer types of resultatives a given predicate may appear with. For example wipe does not entail a result, and allows a range of result XPs for its object as in (38a), as well as resultatives for event participants otherwise not selected for by the verb as in $(38 \mathrm{~b}, \mathrm{c})$. Conversely, break entails a result state and allows a more restricted set of result XPs as in (39a) and does not permit resultatives with unselected objects as in (39b) (data modified from Rappaport Hovav and Levin 1998: 97, (1), 122, (41)).

a. Terry wiped my face (clean/raw). (Normal object, multiple results)

b. Terry wiped the dirt off my face. (Unselected argument)

c. Terry wiped the towel into tathers. (Unselected argument)

(39) a. Kim broke the dishes (to pieces/\#clean).(Normal object, specific result)

b. *Kim broke the dishes off the table/broke his knuckles to the bone. (Unselected argument, unusual result)

The more result the verb entails, the fewer result modifiers are possible and the fewer resultative types are possible. This may explain why consumption predicates behave different from change-of-state predicates. The result state is so heavily lexically specified that the only possible result XPs are those that add what little further specification is possible, ruling out most of the modifiers in (32). The acceptability of up and all gone is because they are the only ones that give an extra level of specificity. In particular, for eat and drink the relevant result state is not technically literal completeness, but rather completeness to a contextually determined degree. The sentence I ate the pizza is acceptable if a few slices are left over, provided the meal is viewed of as "complete" in some sense. But literal complete consumption is more specific, supported by up or all gone, explaining why only these modifiers are even remotely acceptable in (32). Thus (32) does not necessarily argue against an underlying scale of existence, and in fact one is necessary to get the double incremental theme facts that appear to hold for these predicates as well.

\section{Conclusion}

In this paper I argued that homomorphic analyses that preserve mereological properties of incremental themes in the event are crucial to capturing aspectual properties of dynamic predicates that entail change. However, standard analyses fail to capture the fact that all such predicates have multiple incremental themes. I have instead proposed that the relevant semantic property is a ternary $\theta$-relation I refer to as a Figure/Path Relation (FPR). The FPR captures the appropriate properties by making constraints stated on one incremental theme relative to the other and 
vice versa. This analysis, and the effects it is meant to explain, further support the analysis of Beavers $(2002,2006,2008 b, c)$ that all of these dynamic predicates have the same underlying semantics, involving some theme argument transitioning along some scale that describes the change it undergoes in the event. The only difference between all of these predicates is the nature of the scale: for motion predicates it is a physical path, for creation/consumption it is a scale of volume or existence, and for change-of-state it is some property scale. Different verbs simply determine different scales, but the constraints on their mereological properties are identical. In this way we can sweep a disparate set of facts having to do with lexical aspect (as discussed here and in Beavers 2002, 2008b) and argument realization (discussed in Beavers 2006, 2008c) under a single rubric (see also Beavers 2008a for an application of this theory to the notion of affectedness that further solidifies this argument).

\section{References}

Beavers, John: 2002, 'Aspect and the Distribution of Prepositional Resultative Phrases in English'. LinGO Working Paper \#2002-7, CSLI, Stanford University, Stanford.

Beavers, John: 2006, Argument/Oblique Alternations and the Structure of Lexical Meaning, Doctoral Dissertation, Stanford University.

Beavers, John: 2008a, 'On Affectedness'. Unpublished ms., The University of Texas at Austin.

Beavers, John: 2008b, 'Scalar Complexity and the Structure of Events', in J. Dölling, T. Heyde-Zybatow, and M. Schäfer (eds.), Event Structures in Linguistic Form and Interpretation, 245-265. Mouton de Gruyter, Berlin.

Beavers, John: 2008c, 'The Structure of Lexical Meaning: Why Semantics Really Matters'. Unpublished ms., The University of Texas at Austin.

Dowty, David: 1979, Word Meaning and Montague Grammar. Reidel, Dordrecht.

Dowty, David: 1991, 'Thematic Proto-Roles and Argument Selection', Language 67, 547-619.

Filip, Hana: 1999, Aspect, Eventuality Types, and Nominal Reference. Garland, New York.

Garey, Howard B.: 1957, 'Verbal Aspects in French', Language 33, 91-110.

Goldberg, Adele E. and Ray Jackendoff: 2004, 'The English Resultative as a Family of Constructions', Language 80, 532-569.

Hay, Jennifer, Christopher Kennedy, and Beth Levin: 1999, 'Scalar Structure Underlies Telicity in Degree Achievements', in The Proceedings of SALT IX, 127-144.

Jackendoff, Ray: 1996, 'The Proper Treatment of Measuring Out, Telicity, and Perhaps Event Quantification in English', Natural Language and Linguistic Theory 14, 305-354.

Kearns, Kate: 2000, Semantics. St. Martin's Press, New York, NY.

Kennedy, Christopher: 2001, 'Polar Opposition and the Ontology of 'Degrees”, Lin- 
guistics and Philosophy 24, 33-70.

Kennedy, Chris and Beth Levin: 2008, 'Measure of Change: The Adjectival Core of Degree Achievements', in L. McNalley and C. Kennedy (eds.), Adjectives and Adverbs: Syntax, Semantics, and Discourse, 156-182. Oxford University Press, Oxford, UK.

Kennedy, Christopher and Louise McNally: 2005, 'Scale Structure, Degree Modification, and the Semantics of Gradable Predicates', Language 81.

Kiparsky, Paul: 1998, 'Partitive Case and Aspect', in M. Butt and W. Geuder (eds.), The Projection of Arguments: Lexical and Compositional Factors, 265307. CSLI Publications, Stanford.

Krifka, Manfred: 1989, 'Nominal Reference, Temporal Constitution and Quantification in Event Semantics', in R. Bartsch, J. van Benthem, and P. van Emde Boas (eds.), Semantics and Contextual Expressions, 75-115. Foris Publications, Dordrecht.

Krifka, Manfred: 1992, 'Thematic Relations as Links between Nominal Reference and Temporal Constitution', in I. A. Sag and A. Szabolcsi (eds.), Lexical Matters, 29-53. CSLI Publications, Stanford.

Krifka, Manfred: 1998, 'The Origins of Telicity', in S. Rothstein (ed.), Events and Grammar, 197-235. Kluwer, Dordrecht.

Parsons, Terence: 1990, Events in the Semantics of English: A Study in Subatomic Semantics. MIT Press, Cambridge, MA.

Rappaport Hovav, Malka and Beth Levin: 1998, 'Building Verb Meanings', in M. Butt and W. Geuder (eds.), The Projection of Arguments: Lexical and Compositional Factors, 97-133. CSLI Publications, Stanford.

Rothstein, Susan: 2004, Structuring Events. Blackwell, Oxford.

Tenny, Carol: 1987, Grammaticalizing Aspect and Affectedness, Doctoral Dissertation, MIT.

Tenny, Carol: 1992, 'The Aspectual Interface Hypothesis', in I. A. Sag and A. Szabolcsi (eds.), Lexical Matters, 490-508. CSLI Publications, Stanford.

Tenny, Carol: 1994, Aspectual Roles and the Syntax-Semantic Interface. Kluwer, Dordrecht.

Vendler, Zeno: 1957, 'Verbs and Times', The Philosophical Review 66, 143-160.

Verkuyl, Henk J.: 1972, On the Compositional Nature of the Aspects. Reidel, Dordrecht.

Verkuyl, Henk J.: 1993, A Theory of Aspectuality: The Interaction between Temporal and Atemporal Structure. Cambridge Unversity Press, Cambridge, UK.

Wechsler, Stephen: 2001, 'A Fresh Aspect on Resultatives'. Talk given at University of California at Berkeley.

Wechsler, Stephen: 2003, 'Serial Verbs and Serial Motion', in D. Beermann and L. Hellan (eds.), Proceedings of the Workshop on Multi-Verb Constructions. Trondheim.

Wechsler, Stephen: 2005, 'Resultatives under the 'Event-Argument Homomorphism' Model of Telicity', in N. Erteschik-Shir and T. Rapoport (eds.), The Syntax of Aspect, 255-273. Oxford University Press, Oxford. 